\title{
Amyloid pathology arrangements in Alzheimer's disease brains modulate in vivo seeding capability
}

\author{
Claudia Duran-Aniotz 1,2,3,4†, Ines Moreno-Gonzalez ${ }^{1,5,6,7 \dagger}$, Nazaret Gamez ${ }^{1,5}$, Nelson Perez-Urrutia', \\ Laura Vegas-Gomez, ${ }^{5}$, Claudio Soto ${ }^{1,4}$ and Rodrigo Morales ${ }^{1,7^{*}}$ (D)
}

\begin{abstract}
Amyloid- $\beta$ (AB) misfolding is one of the hallmark pathological features of Alzheimer's disease (AD). AD can manifest with diverse symptomatology including variable rates of cognitive decline, duration of clinical disease, and other detrimental changes. Several reports suggest that conformational diversity in misfolded A $\beta$ is a leading factor for clinical variability in $A D$, analogous to what it has been described for prion strains in prion diseases. Notably, prion strains generate diverse patterns of misfolded protein deposition in the brains of affected individuals. Here, we tested the in vivo prion-like transmission features of four AD brains displaying particular patterns of amyloidosis. AD brains induced different phenotypes in recipient mice, as evaluated by their specific seeding activity, as well as the total amount of $A \beta$ deposited surrounding vascular structures and the reactivity of amyloid pathology to thioflavin S. Our results support the notion that AD-subtypes are encoded in disease-associated A . Further research exploring whether AD include a spectrum of different clinical conditions or syndromes may pave the way to personalized diagnosis and treatments.
\end{abstract}

Keywords: Alzheimer's disease, Amyloid-beta, Prions, Strains, Pathology

\section{Introduction}

Alzheimer's disease (AD) is a heterogeneous and multifactorial brain disorder and the most common form of dementia in elderly population. AD is characterized by progressive neuronal degeneration, leading to severe cognitive impairments $[1,2]$. The most prominent pathological hallmarks of $\mathrm{AD}$ are the extracellular deposition of amyloid- $\beta$ (A $\beta)$ peptides in the form of plaques and the intracellular accumulation of hyper-phosphorylated Tau (p-Tau) protein as neurofibrillary tangles [3]. A $\beta$, the proteolytic product of a transmembrane protein known as amyloid precursor protein (APP), is attributed with

\footnotetext{
*Correspondence: Rodrigo.MoralesLoyola@uth.tmc.edu

${ }^{\dagger}$ Claudia Duran-Aniotz and Ines Moreno-Gonzalez have contributed equally to this work.

${ }^{1}$ Department of Neurology, The University of Texas Health Science Center at Houston, 6431 Fannin, St. Houston, TX 77030, USA

Full list of author information is available at the end of the article
}

leading roles in AD as mutations in APP [4] or proteins linked to its proteolytic processing are associated with disease inheritance $[5,6]$. In addition, strong evidence suggest that the misfolding and deposition of $A \beta$ leads to the accumulation of neurotoxic hyper-phosphorylated tau [7]. Other important AD lesions, such as loss of neurons, synapse disruption, axonal trafficking impairment, oxidative stress [8], and activation of chronic neuroinflammation [9] are also associated with pathogenic $A \beta$.

$\mathrm{AD}$ is clinically and pathologically diverse. Variable clinical features include age at disease onset, clinical duration of the disease, rate of cognitive decline, manifestation of motor impairments, among others [10]. Pathologically, $A \beta$ accumulation follows a sequential/ progressive anatomical distribution that is initiated in the neocortex and subsequently expands to allocortical regions, striatum, brain stem and cerebellum [11]. Regardless of this well-conserved deposition

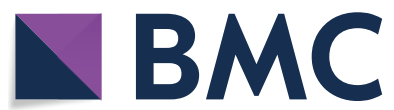

(c) The Author(s) 2021. Open Access This article is licensed under a Creative Commons Attribution 4.0 International License, which permits use, sharing, adaptation, distribution and reproduction in any medium or format, as long as you give appropriate credit to the original author(s) and the source, provide a link to the Creative Commons licence, and indicate if changes were made. The images or other third party material in this article are included in the article's Creative Commons licence, unless indicated otherwise in a credit line to the material. If material is not included in the article's Creative Commons licence and your intended use is not permitted by statutory regulation or exceeds the permitted use, you will need to obtain permission directly from the copyright holder. To view a copy of this licence, visit http://creativecommons.org/licenses/by/4.0/. The Creative Commons Public Domain Dedication waiver (http://creativeco mmons.org/publicdomain/zero/1.0/) applies to the data made available in this article, unless otherwise stated in a credit line to the data. 
pattern, extracellular $\mathrm{A} \beta$ deposits can manifest in diverse arrangements, including neuritic cored plaques, diffuse (lake-like and fleecy deposits), cotton wool plaques, etc., in addition to cerebral amyloid angiopathy (CAA) $[12,13]$. Also, A $\beta$ deposits can be differentially distributed at cellular (intra-, extra-) or tissue (parenchyma or vessels) levels $[14,15]$. Unfortunately, the contribution of each type of deposits to neuronal death, brain inflammation and $\mathrm{AD}$ phenotypes is not completely understood. The specific distribution of these lesions across AD patients are thought to play a key role in brain atrophy and may modulate the clinical manifestations observed across individuals afflicted by $\mathrm{AD}[16,17]$.

Currently, the factors or events directing $A \beta$ to deposit in specific patterns are not known. Various lines of evidence suggest that $A \beta$ misfolds and spreads in AD brains following mechanisms that resemble the replication of infectious prion proteins $\left(\mathrm{PrP}^{\mathrm{Sc}}\right)[18-21]$. In that sense, several prion-like properties have been attributed to $\mathrm{A} \beta$, including conformation and dose-dependent propagation [22-24], and brain invasion after administering preformed aggregates by different routes [25-27]. An intriguing feature of prions involves their conformational "strain" diversity. Compelling evidence suggest that prions strains differ in the conformational arrangements that the constituents prion proteins acquire [28, 29]. Strain-specific prion infection may lead to clinically diverse diseases as observed in experimental animals $[30,31]$ and humans [32-34]. Prion strains also induce variable distribution of $\mathrm{PrP}^{\mathrm{Sc}}$ and patterns of spongiform degeneration in the brain of diseased individuals [33, 3537]. Some reports suggest that such variability also exist for misfolded $A \beta$ peptides [15, 38-42]. Actually, conformational variability on $\mathrm{AD}$-associated $\mathrm{A} \beta$ has been linked to rapid disease progression [43]. Experimental data demonstrate that misfolded $A \beta$ aggregates propagate different structural motifs in susceptible mouse models, partially resembling bona fide strain-specific prion transmission [39-41]. Regardless of this evidence, additional research is needed to establish whether strain variation at the level of $A \beta$ modulates AD clinical and pathological variability.

In this study, we analyzed the in vivo prion-like propagation of disease-associated $\mathrm{A} \beta$ from $\mathrm{AD}$ brains displaying different amyloid pathology. Our results show that these $\mathrm{AD}$ brains induce diverse pathological features in treated mice, as measured by the intrinsic ability of each AD brain to promote amyloid pathology (seeding activity), and the particular vascular and thioflavin $S$ positive pathology they induced. Our results suggest that different arrangements of misfolded $A \beta$ template the propagation of unique pathological features associated with $\mathrm{AD}$.

\section{Materials and methods}

\section{Human tissues}

Brain samples (frontal cortex) used in this study were collected post mortem from four clinically diagnosed $\mathrm{AD}$ patients and one non-demented individual. Left hemisphere was frozen and used to generate the inoculum. The right hemisphere was fixed in formaldehyde $37 \%$. Both brain areas were dissected in their different anatomical components. Dissected fixed tissue was processed in increasing graded ethanol, embedded in paraffin and sliced. Specific information from each donor is provided in Table 1.

\section{Transgenic mice}

APP/PS1 transgenic mice were used in this study. These mice over-express the human version of amyloid precursor protein (APP) harboring the Swedish double mutation (K670M and N671L) and the human presenilin-1 protein with the deltaE9 mutation (PSEN1- $\triangle E$ 9) [44]. Treated animals were housed in groups of up to 5 in individually ventilated cages under standard conditions $\left(22{ }^{\circ} \mathrm{C}, 12 \mathrm{~h}\right.$ light-dark cycle) receiving food and water ad libitum. 4-6 animals per experimental group were used as indicated in each section. Males and females were indistinctly used (overall $42 \%$ males, 58\% females). All animal experiments were carried out in accordance with the National Institutes of Health regulations and IACUC guidelines and approved by the committee of animal use for research at the University of Texas Health Science Center at Houston.

\section{Preparation and characterization of human brain inocula}

Frozen frontal cortex samples were homogenized using an automatic homogenizer (Precellys 24-dual, Bertin Instruments) at $10 \%(\mathrm{w} / \mathrm{v})$ in PBS containing a cocktail of protease inhibitors (Roche Diagnostics $\mathrm{GmbH}$ ). Resulting homogenates were stored at $-80{ }^{\circ} \mathrm{C}$ until used for animal injection or ELISA measurements of $A \beta$ peptides (as explained below).

Table 1 Relevant information of brain donors

\begin{tabular}{lllll}
\hline Code & Sex & $\begin{array}{l}\text { Age } \\
(y / 0)\end{array}$ & Diagnosis & Cause of death \\
\hline 60129 & Male & 84 & AD & Probable myocardial infarction \\
60068 & Female & 69 & AD & Pulmonary \\
60649 & Male & 65 & AD & Alzheimer disease \\
51486 & Female & 79 & AD & Renal failure \\
58652 & Male & 59 & Non-demented & Cardiopulmonary arrest
\end{tabular}




\section{Intra-cerebral inoculation of brain extracts}

$\sim 30$ days-old APP/PS1 mice were intra-cerebrally injected with $10 \mu \mathrm{L}$ of $10 \%(\mathrm{w} / \mathrm{v})$ brain homogenates from AD patients or a non-demented individual. For injection, animals were anesthetized using isoflurane and fixed to a mouse stereotaxic frame. Unilateral injections were performed at a single point in the right hippocampal area using the following coordinates as measured from Bregma: antero- posterior (AP), $-1.8 \mathrm{~mm}$; mediolateral (ML), $-1.8 \mathrm{~mm}$; dorso-ventral (DV), $-1.8 \mathrm{~mm}$. Immediately after treatment, skin was closed using surgical suture. The injection was conducted at a rate of 0.5 $\mu \mathrm{L} / \mathrm{min}$ and the needle was left in place for $3 \mathrm{~min}$ before retraction. Animals were placed on a thermal pad until recovery and monitored daily for several days. Mice were sacrificed by $\mathrm{CO}_{2}$ inhalation at $\sim 150$ days after inoculation. Brains were removed and stored in $10 \%$ formalin for histological studies.

\section{Histological analysis}

$10-\mu \mathrm{m}$-thick serial slices from all animal groups or human subjects were processed in parallel for histological analyses. Mouse-derived specimens were processed from lambda 0 to lambda $-4 \mathrm{~mm}$. For immunohistochemistry, sections were deparaffinazed and hydrated and the endogenous peroxidase activity was blocked with $3 \% \mathrm{H}_{2} \mathrm{O}_{2} / 10 \%$ methanol in PBS for $20 \mathrm{~min}$. After formic acid epitope retrieval (formic acid $85 \%$ for $5 \mathrm{~min}$ ), primary antibody 4G8 was incubated over night at a 1:1,000 dilution at room temperature (Covance, Princeton, NJ). HRP-linked secondary sheep anti-mouse antibody at a 1:1,000 dilution (GE Healthcare, Little Chalfont, UK) was incubated for $2 \mathrm{~h}$ at room temperature. Peroxidase reaction was visualized using DAB Kit (Vector, Burlingame, CA) following the manufacturer's instructions. Finally, sections were dehydrated in graded ethanol, cleared in xylene, and cover-slipped with DPX mounting medium (Innogenex, San Ramon, CA). Additional brain sections were incubated with Thioflavin-S (ThS) solution $(0.025 \%$ in $50 \%$ ethanol) for $8 \mathrm{~min}$, and coverslipped with DPX. All samples were analyzed using an inverted microscope for bright field and an epifluorescent microscope for ThS staining (DMI6000B, Leica, Buffalo Grove, IL, USA) and then quantification analysis was performed using the ImagePro software (Rockville, MD, USA). Five tissue slices per animal/staining, taken every 10 slices were used for image analysis quantifications. For specific quantification of vascular $A \beta$ deposition, slices stained with 4G8 were quantified for brain-parenchymal blood vessel associated signals as described before [45] in the cerebral cortex. Leptomeningeal deposits were not included in these analyses. $A \beta$ and ThS burdens were defined as the area of the brain labeled per the total area analyzed. Burden quantification was performed by an investigator blinded to the experimental groups. Vascular deposition in the hippocampus of treated mice was scarce and was semi-quantitatively evaluated in a present/absent manner (Additional file 1: Table 1).

For double staining, human sections were auto-fluorescence blocked with Autofluorescence Eliminator Reagent (Millipore 2160), subjected to antigen retrieval using $10 \mathrm{mM}$ citrate buffer $\mathrm{pH} 6.0$ for $30 \mathrm{~min}$ at $80^{\circ} \mathrm{C}$ and blocked with 5\% BSA to avoid non-specific binding. Thereafter, samples were incubated with rabbit antismooth muscle actin (SMA) (Proteintech, Illinois, USA) primary antibody overnight at a 1:1,000 dilution at room temperature. Consecutively, sections were incubated with mouse anti-4G8 antibody (as stated previously) or ThS solution $(0.025 \%$ in PBS for $8 \mathrm{~min})$. Primary antibodies were visualized with anti-mouse Alexa Fluor488 and anti-rabbit Alexa Fluor 594 at a 1:1,000 dilution for $1.5 \mathrm{~h}$ (ThermoFisher, USA). Finally, samples were washed and cover-slipped with FluorSave mounting medium (Millipore Sigma, Massachusetts, USA). Analyses of vascular amyloidosis in mice's brains were done as explained above but using 4G8 antibody instead of ThS.

\section{ELISA quantification of $A \beta$ species}

Brain extracts from AD patients and a non-demented individual were subjected to a previously described serial A $\beta$ extraction protocol [22, 46, 47]. Briefly, 10\% (w/v) brain homogenates were centrifuged in L100K ultracentrifuge tubes (Beckman-Coulter, Brea, CA) at 32,600 rpm for $1 \mathrm{~h}$ at $4{ }^{\circ} \mathrm{C}$, using a $42.2 \mathrm{Ti}$ rotor. Supernatants were saved (PBS fractions) and pellets were resuspended in $2 \%$ sodium dodecyl sulfate (SDS) by pipetting and sonication (using a bath sonicator) until complete disruption. Samples were subjected to the same centrifugation procedure explained above. The resulting supernatants were collected (and saved as SDS fractions), pellets resuspended in $70 \%$ formic acid (FA, Fisher Scientific, Waltham, MA) and sonicated in a bath sonicator until complete disruption. Then, samples were centrifuged for $30 \mathrm{~min}$ using the same conditions explained above and supernatants were collected (FA fractions). FA fractions were 20-fold diluted on $1 \mathrm{M}$ Tris buffer, pH 11 (Sigma-Aldrich, St. Louis, MO) to neutralize $\mathrm{pH}$. $\mathrm{A} \beta_{42}$ peptides present in these samples were measured by ELISA using a commercially available kit (KHB3442, Invitrogen, Carlsbad, CA). ELISA was performed following manufacturer's instructions.

\section{Calculation of seeding activity ratio}

Seeding activity ratio for each inoculum was calculated by dividing burden values present in each animal (cortex 
and hippocampus) by the $\mathrm{A} \beta$ burden present in each $\mathrm{AD}$ brain used for inoculation.

\section{Comparisons between different pathological features in treated mice}

Each parameter measured in mice injected with $\mathrm{AD}$ brain extracts (A $\beta$ burden and ThS burden in different regions, vascular amyloidosis and induction ratios) were normalized to a value of 1 considering the maximum averaged value among all groups for each parameter. Data obtained in this manner for all pathological assessments was combined in a single graph using Microsoft Excel.

\section{Statistical analyses}

Data are expressed as means \pm standard error of the mean (SEM). After confirming normal distribution with Skewness/Kurtosis statistic test, one way ANOVA was used to analyze differences in histological assays. Statistical analyses were performed using Graph Pad Prism 5.0 software. Statistical differences were considered significant for values of $p<0.05$.

\section{Results}

\section{Characterization of $A \beta$ pathology in the brains of four $A D$} patients

$\mathrm{A} \beta$ pathology in the brains of $\mathrm{AD}$ patients was assessed by histopathological and biochemical analyses. All four patients included in this study (AD60129, AD60068, AD60649 and AD51486) were clinically diagnosed for AD. Immunohistochemical staining against $A \beta$ revealed strikingly different patterns of amyloid deposition among them (Fig. 1). Brain AD60129 displayed abundant neuritic and cored plaques and some diffuse aggregates (Fig. 1a). As expected, many of the deposits present in this sample were reactive against Thioflavin S (ThS, Fig. 1e), a dye binding compact amyloid structures [48]. AD60068 showed small intracellular aggregates but no parenchymal deposits were observed (Fig. 1b). This feature was unique among all specimens used in this study. No ThS-positive deposits were found in this brain tissue (Fig. 1f). AD60649 sample displayed abundant A $\beta$ deposition albeit the majority of aggregates observed in this patient were less compact as the ones observed in sample AD60129 while also displaying abundant diffuse $A \beta$ plaques (Fig. 1c). Here, ThS positive signals were observed in smaller but more abundant deposits (Fig. 1g). Finally, brain AD51486 displayed diffuse (cotton wool, Fig. 1d) and cored $A \beta$ deposits with abundant cerebral amyloid angiopathy (CAA). The presence of CAA in this and all other $\mathrm{AD}$ samples was further characterized by immunohistochemical (IHC) analyses. Specifically, ThS positive $A \beta$ deposits were assessed for co-localization with the smooth muscle actin (SMA) protein, a marker of blood vessels. Panels I-L in Fig. 1, and Additional file 1: Fig. 1, further confirm the strong CAA phenotype in sample AD51486 compared to all other specimens used in this study. Additional file 1: Fig. 1 additionally shows that occasional CAA was found only in sample AD60649 (a single event in all tissue slices analyzed). Vascular amyloidosis was absent in AD60129 and AD60068 brains. ThS positive amyloidosis in AD51486 was observed for both, parenchymal and vascular aggregates (Fig. 1h).

$A \beta$ burden quantification of 4G8-inmunoreactivity by image analysis (Fig. $1 \mathrm{~m}$ ) showed similar values for samples AD60129, AD60068 and AD51486. Amyloid deposition in sample AD60068, displaying abundant intracellular $A \beta$ accumulation, was considerable lower ( ninefold) compared to all other samples. These results were confirmed by ELISA measurements of $A \beta_{40}$ (Fig. 1n) and $A \beta_{42}$ (Fig. 1o) after serial extraction in PBS, SDS and FA. $A \beta_{40}$ levels measured in the FA-fractions were substantially higher in sample AD51486 compared to all other specimens, in line with the higher vascular amyloidosis observed for this sample in IHC analyses (Fig. 11). Brain AD60649 also displayed high $\mathrm{A} \beta_{40}$ concentrations in the SDS- and FA- fractions. However, values collected in the SDS-fraction were not different compared to the ones in AD60129, AD60068 and the brain of a nondemented individual (sample 58652). As expected, levels of PBS-insoluble $A \beta_{42}$ proteins displayed higher differences between AD specimens and the control brain. In agreement with the SDS- and FA- extracted $A \beta_{40}$ levels, samples AD51486 and AD60649 contained the highest accumulation of this disease-associated peptide $(\sim 3-7$ times) compared to sample 58652 (aged non-demented control). Sample AD60129 displayed lower PBS-insoluble $\mathrm{A} \beta_{42}$ concentrations compared to AD51486 and AD60649, although substantially higher compared with the control brain. In contrast, sample AD60068 did not show $A \beta_{42}$ concentrations that differed from the nondemented control brain. Overall, the PBS-soluble $A \beta_{40}$ and $A \beta_{42}$ levels were not substantially different across all samples analyzed. A summary of the findings described in Fig. 1 is presented in Table 2.

The characterization presented above is a good example of the pathological variability displayed across the brains of $A D$ patients. In that sense, extracts prepared from these AD tissues were used to test their intrinsic seeding activities in susceptible mouse models of cerebral amyloidosis.

\section{In vivo seeding activity of $A D$ brains displaying different amyloid pathology}

Extracts from AD brains displayed in Fig. 1 were intracerebrally inoculated in $\sim 30$ days old APP/PS1 mice. Animals were sacrificed 150 days after the treatment and 

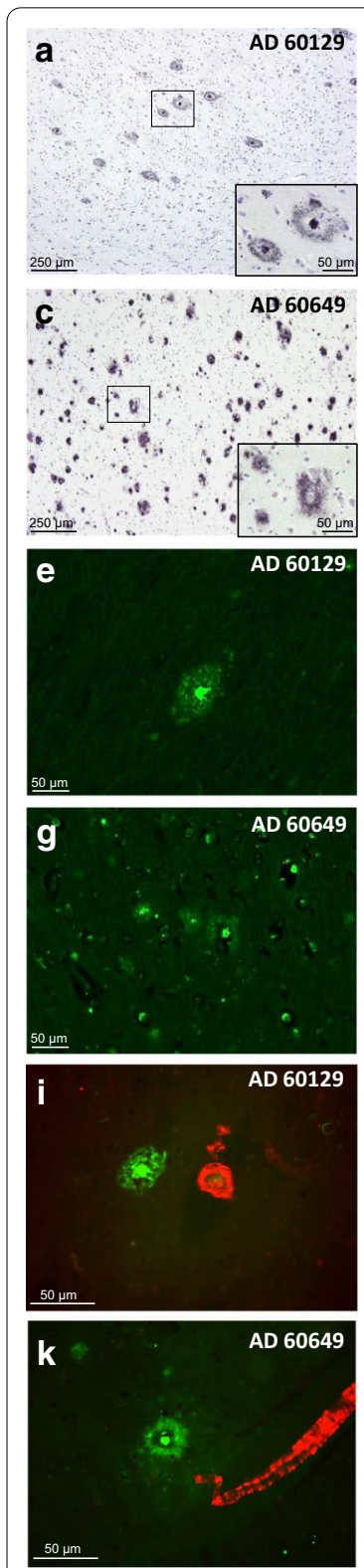

\section{b}
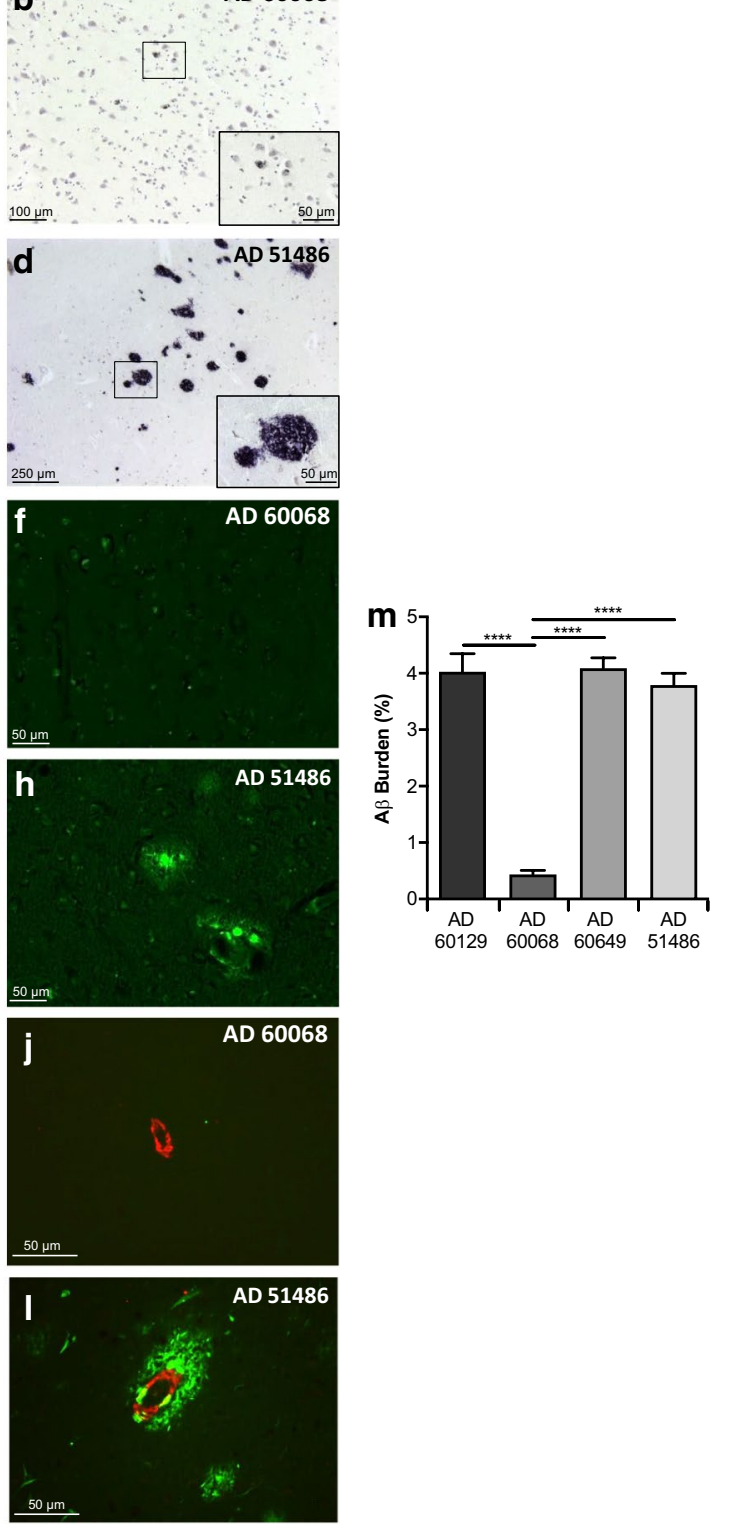

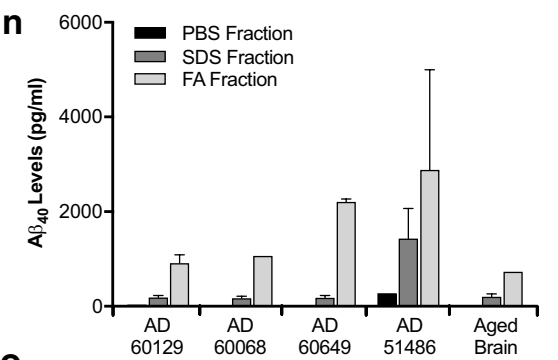

$\mathbf{0}$

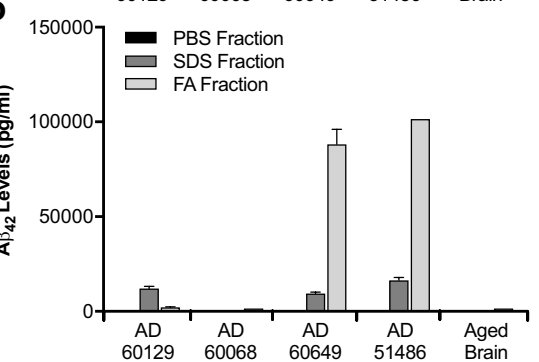

Fig. 1 Characterization of four AD brains displaying different amyloid pathology. Brains from four clinically confirmed AD patients were characterized for A 3 deposition using immunohistochemistry and ELISA. a-d Brain slices from patients AD60129, AD60068, AD60649 and AD51486 were immunostained to assess $A \beta$ deposition. Insets represent higher magnifications of representative amyloid plaques in each specimen. $\mathbf{e}-\mathbf{h}$ Thioflavin S (ThS) staining for the same samples described above. Characterization for brain 58652 (non-demented) is provided in [46]. g-j Double staining using ThS (green) and anti-SMA antibody (red) was performed to study the presence of vascular A $\beta$ in human samples. (M) Image analysis quantification (\% burden) of $A \beta$ pathology in samples AD60129, AD60068, AD60649 and AD51486. (N-O) $A \beta_{40}$ and $A \beta_{42}$ was serially extracted in PBS, SDS and FA as explained in Materials and Methods. A $\beta$ peptides in each fraction were measured by human-specific A ELISA kits. Data values were expressed as mean \pm SEM. Data from (M) was obtained from multiple tissue slices and analyzed by one-way ANOVA followed by Tukey's multiple comparison post-test. ${ }^{*} p<0.05,{ }^{* *} p<0.01,{ }^{* * *} p<0.001,{ }^{* * *} p<0.0001$

brains collected for histopathological analyses (Fig. 2). Untreated animals (Fig. 2a, g) and mice injected with brain extract from an aged non-demented individual $(58,652)$ (Fig. 2b, h) acted as negative controls. Brain slices from mice in the control groups displayed modest amyloid pathology, similarly as previously described $[46,49]$. Image-analysis quantification of $A \beta$ burden in cortex and hippocampus of mice in these two negative control groups did not display significant differences, suggesting that the non-demented brain does not carry 
Table 2 Summary of pathological features observed across AD patients

\begin{tabular}{llllll}
\hline Code & A deposits & A burden & Vascular $A \beta$ & Insoluble $A \beta_{40}$ & Insoluble $A \beta_{42}$ \\
\hline 60129 & Cored plaques & High & Low & Low & Medium \\
60068 & Intracellular & Low & None & Low & Low \\
60649 & Diffuse and cored plaques & High & Low & Medium & High \\
51486 & Diffuse (cotton wool) & High & High & High & High \\
58652 & ND & ND & ND & Low & Low
\end{tabular}

ND notdetermined

$A \beta$ seeding activity (Fig. $2 m, n)$. On the contrary, most AD brains (AD51486, AD60649 and AD60068) induced significantly higher amyloid pathology compared to the control groups. Brain AD60129 displayed a positive trend in terms of amyloid deposition compared to controls, although this increase did not reach statistical significance. Amyloid deposition of mice treated with $\mathrm{AD}$ brains was arranged in the following pattern: AD51486 > AD60649 > AD60068 > AD60129.

In order to explore the intrinsic seeding activity of each $\mathrm{AD}$ brain, burden values present in each animal (cortex and hippocampus) were divided by the $A \beta$ burden present in each corresponding AD brain used for inoculation (Fig. 2o). Surprisingly, the highest intrinsic seeding activity was found for brain AD60068, the one displaying the lowest $A \beta$ accumulation burden. Using the same analysis, all other brains showed lower but similar seeding activities among themselves. These results show that amyloid seeding is independent of the total $A \beta$ content, but a consequence of their specific pathological arrangements.

\section{Inoculum-dependent induction of vascular amyloid deposition}

Misfolded $\mathrm{A} \beta$ in $\mathrm{AD}$ can deposit in either the brain parenchyma or vasculature $[14,15]$. CAA leads to deleterious events including microhemorrhages that may worsen clinical deterioration $[50,51]$. Previous results show that exogenous $A \beta$ seeding induce CAA in susceptible mice $[25,52]$. Considering the variable vascular amyloidosis in the four AD samples included in this study, we assessed whether they differentially induced CAA in treated mice. When present, most vascular aggregation was found in the cerebral cortex (Fig. 3a-f). As expected, no vascular aggregates were observed in blood vessels of the brain parenchyma in untreated APP/ PS1 mice, as these animals develop few CAA events only at advanced ages when brain amyloidosis is extensive (unpublished data). Surprisingly, the brain from the aged non-demented control individual induced some CAA in recipient mice (Fig. $3 \mathrm{~b}$ ). This result was unexpected, as the total $A \beta$ burden in the brain of these mice was not significantly different compared to untreated controls.
However, it is important to highlight that this inoculum had detectable levels of insoluble $A \beta$ (Fig. 1n) that could alter some aspects of $A \beta$ misfolding propagation in recipient mice. All AD samples, with the sole exception of AD60068 (displaying small distribution of mostly intracellular aggregates) induced vascular amyloidosis in blood vessels of the brain parenchyma of recipient mice (Fig. 3). Vascular amyloidosis in the AD60649- and AD51486-derived groups was significantly higher compared to the one found in mice receiving the control injectate and ranged from 23.7 to $19.4 \%$ of the total deposition in the brain cortex (Fig. 3g). These results are relevant, considering that the AD60068 homogenate displayed the highest intrinsic seeding capacity, and suggest that $A \beta$ extracellular deposits may modulate the propagation of amyloidosis to vascular structures.

We also evaluated $A \beta$ deposition in hippocampal blood vessels of all treated mice. CAA in this specific brain region was scarce and it was found in just a fraction of experimental mice (Additional file 1: Table 1). The presence of vascular amyloidosis in this brain region largely correlated with cortical values as it was found only in some animals treated with the AD60649 and AD51486 inocula. No vascular $A \beta$ deposits were found for animals injected with the AD60129 brain extract, despite this injectate promoted vascular pathology in the cortex. This data further suggest that induction of brain amyloidosis is not linear and depends of the intrinsic properties of each inoculum.

\section{Prion-like induction of amyloid pathology induces diverse populations of $A \beta$ deposits}

Compact amyloid aggregates can react to several dyes, such as thioflavin and Congo red [48]. For that reason, these reagents have been extensively used as pathological markers in both diagnostic and research contexts. Importantly, the degree of compactness displayed by amyloid aggregates can be used as a surrogate of pathological variability. We stained brain tissue of experimental and control mice with ThS and compared their pathological phenotypes at this level. Our results show that all groups 
displayed ThS positive $A \beta$ deposits in both cortex and hippocampus (Fig. 4a-l). Cortical measurements showed a heterogeneous ThS-positive burden, albeit no significant differences were found across the groups (Fig. $4 \mathrm{~m}$ ). In the hippocampus, sample AD51486 induced significantly higher levels of ThS-positive deposits, in line with the highest overall amyloid induction generated by this particular sample (Fig. 4n).

Considering the different levels of total amyloidosis observed across the groups, we calculated the ratio between ThS values and 4G8-positive IHC to assess the distribution of plaques reactive to ThS compared to the total $\mathrm{A} \beta$ deposition present in each brain (Fig. 4o, p). We observed that most of the deposits found in untreated control mice were reactive against ThS. On the contrary, the ThS/4G8-reactivity ratios in animals inoculated with the $\mathrm{AD}$ and control brains were significantly lower in the hippocampus (Fig. 4o). In the cortex, all AD-inoculated brains exhibited different values compared to the untreated control, while the group induced with the control brain $(58,652)$ was not significantly different, in line with vascular data displayed in Fig. 3. This data further suggests that brains from AD patients displaying differential pathology propagate inoculum-specific deposits in susceptible mice.

\section{Discussion}

$\mathrm{AD}$ is linked to several pathological features, including $A \beta$ and tau deposition, glial activation and brain atrophy [2]. Variations in some of these properties have been associated with clinical manifestations [15]. Considering $\mathrm{A} \beta$ misfolding and accumulation as an early event in $\mathrm{AD}$, disparities at this level may be amplified downstream at pathological cascades resulting in various clinical symptoms [15-17].

Conformational strain diversity has been extensively described for infectious prions [29, 53, 54]. Rodentadapted prion strains are known to generate distinguishable changes in terms of prion protein deposition and spongiform degeneration in the brain $[29,31,36,37$, 54]. Prion strains can be discriminated at the molecular level by assessing several parameters such as their electrophoretic mobility after protease digestion, glycosylation profiles, and resistance to proteolytic digestion or denaturation [29, 55-57]. Similar characterizations on misfolded $A \beta$ aggregates suggest that this diseaseassociated protein also displays conformational strain variations $[39,43]$. The existence of "A $\beta$ strains" has been attributed as the cause of rapid and slow cognitive decline observed across AD patients [43]. Structural changes have also been found in misfolded $A \beta$ structures from patients afflicted by different AD types [58]. A $\beta$ strains are able to propagate various conformations in in vitro and in vivo systems $[39,42,58]$. However, the biological significance of misfolded strain variation in AD's $A \beta$ is not well understood.

As mentioned, prion strains accumulate in the brain in different patterns, displaying variable anatomical tropisms and deposits, including diffuse, compact, extra- and intra-cellular inclusions [53]. Similar variation in $A \beta$ deposition has been described in $\mathrm{AD}[14,15]$. Here, we characterized amyloid deposition in the brain of four individuals clinically diagnosed with AD dementia. These patients showed strikingly different patterns of amyloid deposition in terms of total $A \beta$ levels, cellular location, and type of aggregates (Fig. 1 and Table 2). To partially assess the pathological significance of this assortment of aggregates, we inoculated brain extracts from these patients in susceptible mice $[46,49]$. Interestingly, the extent of amyloid induction was not directly associated with the levels of $A \beta$ administered in each case (Fig. 2). In fact, the brain displaying the lowest net amount of $A \beta$ deposits (AD60068) was the one with the highest seeding activity (Fig. 20). Patient AD60068 displayed small intracellular inclusions that were less compact compared to the ones observed in the other AD brains included in this study. Whether this sample is enriched in oligomeric $A \beta$ species, generally considered as the best seeds [59], will be reported in future studies. In addition, we observed that $A \beta$ pathology in the brains of experimental mice displayed different arrangements as judged by their differential reactivity to ThS (Fig. 4) and tropism to blood vessels (Fig. 3). This additional data set suggests that the pathological information encoded in $A \beta$ seeds is able to propagate different pathological traits. The pathological features generated in treated mice are summarized in Fig. 5. There, it can be clearly appreciated that the four different $\mathrm{AD}$ inocula promote the appearance of different patterns of brain amyloidosis, probably attributed to the

\footnotetext{
(See figure on next page.)

Fig. 2 A $\beta$ seeding activity of pathologically diverse AD brains. Brain cortex $(\mathbf{a}-\mathbf{f})$ and hippocampus $(\mathbf{g}-\mathbf{I})$ of mice inoculated with different brain extracts were analyzed for their content of $A \beta$ deposits. Equivalent specimens from untreated mice were included as controls. $\mathbf{m}, \mathbf{n} I m a g e$ analysis quantification (\% burden) of A $\beta$ pathology in cortex $(\mathbf{m})$ and hippocampus (n). o The intrinsic seeding capacity of each inoculum was calculated as the ratio between $A \beta$ burden in each mouse and the burden in the inoculum used for treatment. $N=4-6 / g r o u p$. Data value were expressed as mean \pm SEM. Data from M and N were analyzed by Student's t-test comparing the experimental groups with Aged Brain. Data from o were analyzed by one-way ANOVA followed by Tukey's multiple comparison post-test. ${ }^{*} p<0.05,{ }^{* *} p<0.01,{ }^{* * *} p<0.001$
} 


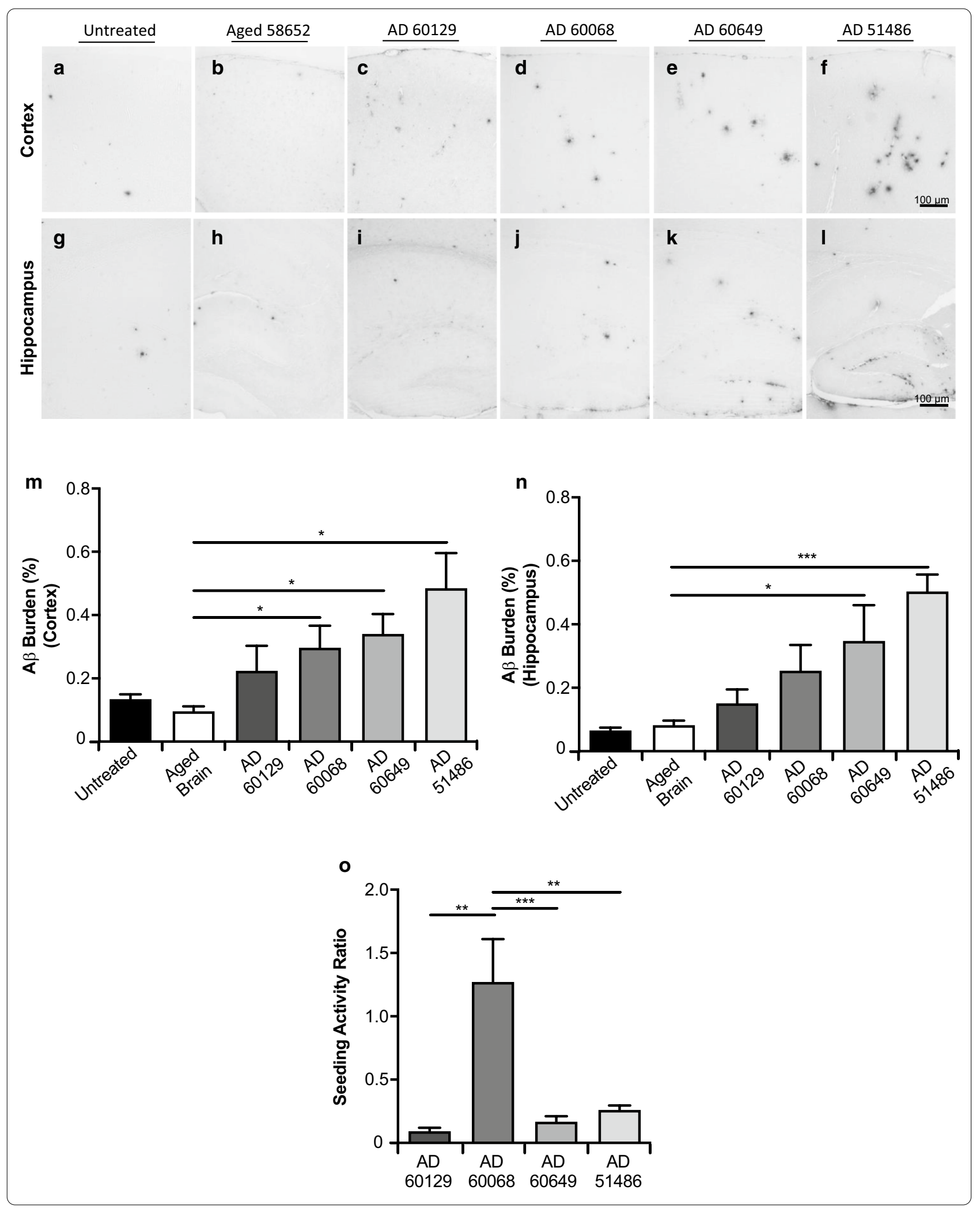



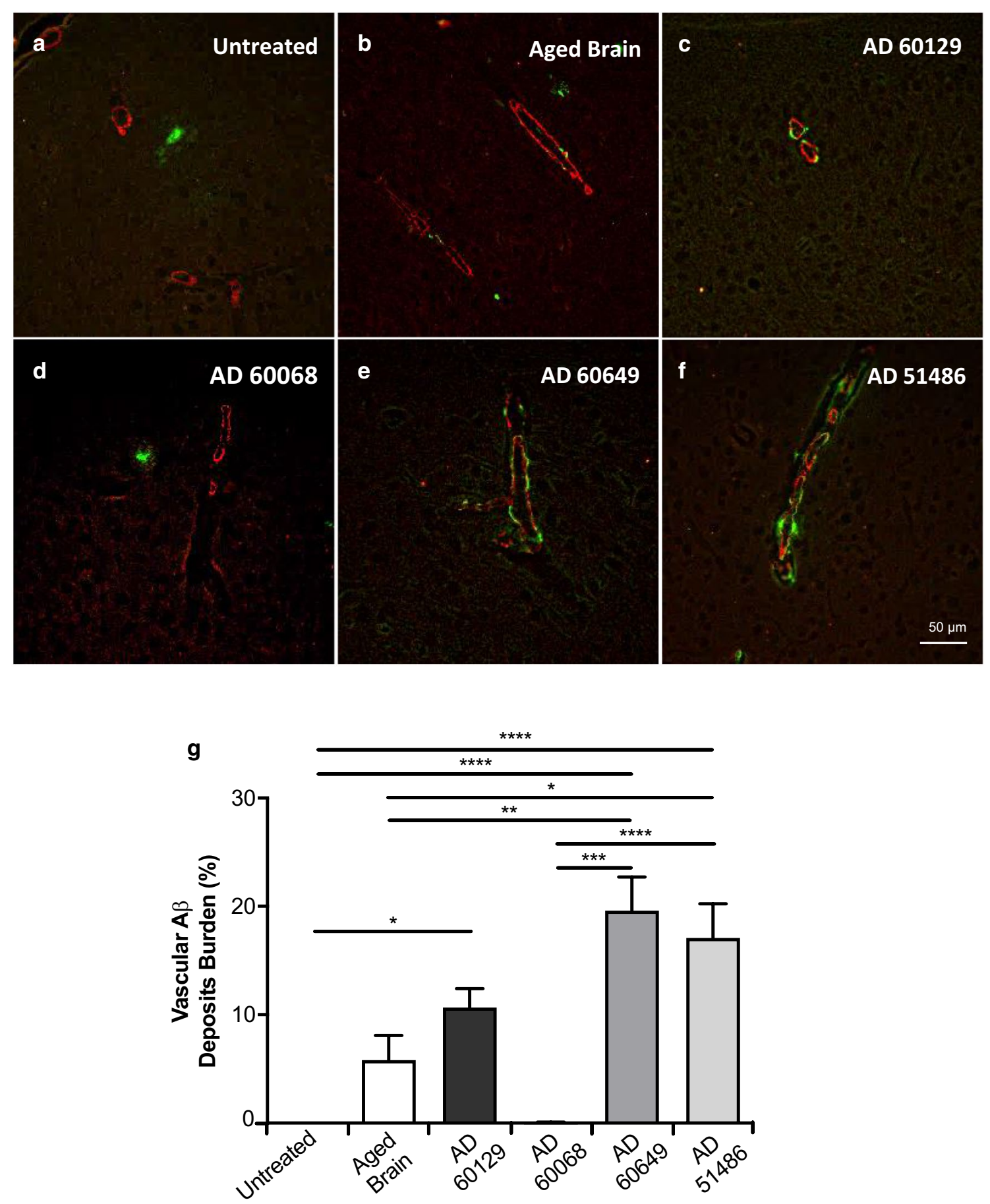

Fig. 3 Vascular deposition in the brain of APP/PS1 mice challenged with AD brain extracts. a-f Double immunofluorescence staining for A (green) and SMA (red) in mice's brains inoculated with human AD brain extracts. $\mathbf{g}$ The extent of amyloid deposition associated with vessels was calculated as previously reported [45] using bright field images. Values for each group are expressed as the area of A 4 4G8-inmunoreactivity in vascular structures divided by the total $A \beta$ burden and multiplied by $100 . N=4-6 /$ group. Data value were expressed as mean $\pm S E M$. Data were analyzed by one-way ANOVA followed by Tukey's multiple comparison post-test. ${ }^{*} p<0.05,{ }^{* *} p<0.01,{ }^{* * *} p<0.001,{ }^{* * * *} p<0.0001$ 


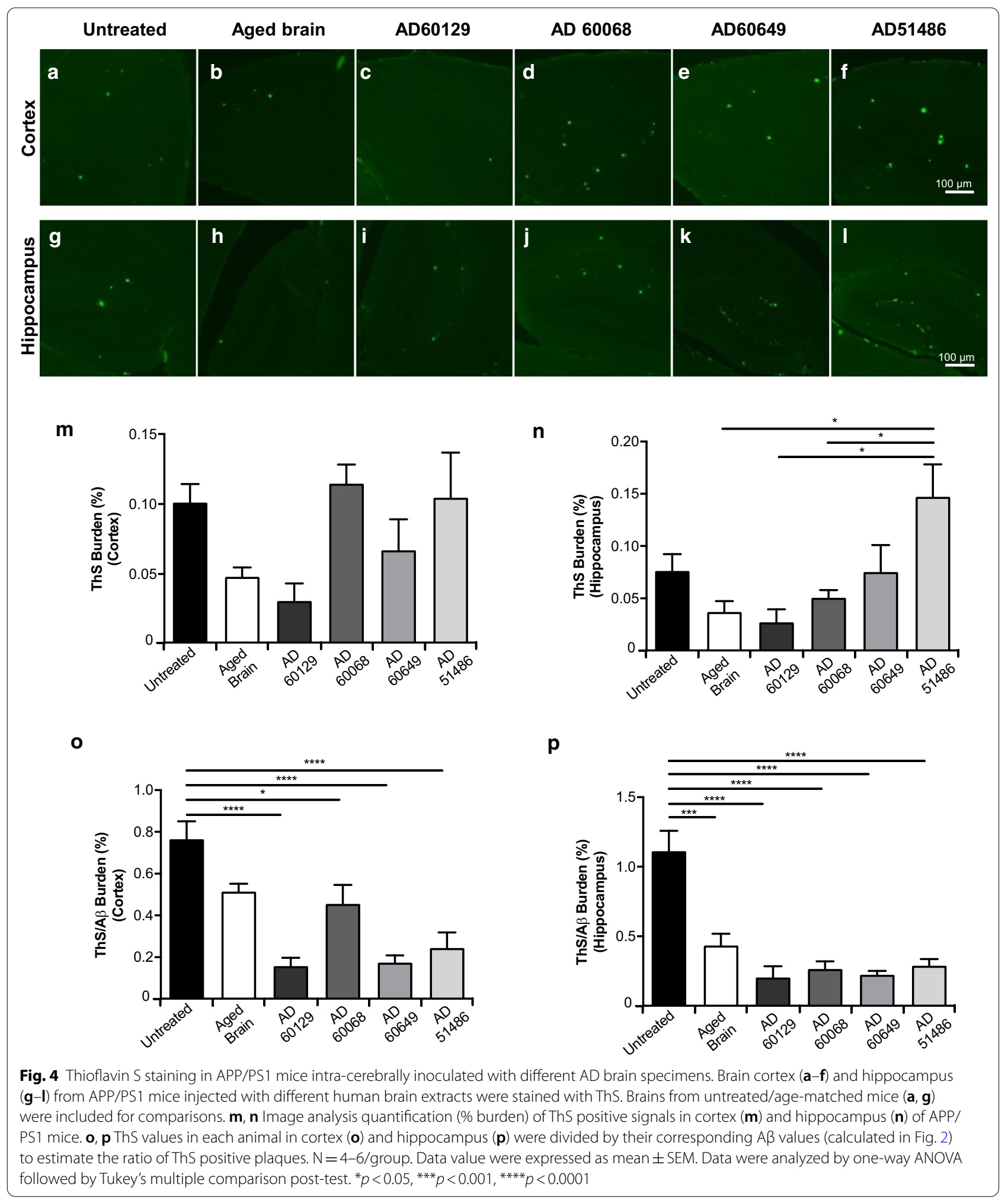

different arrangements of amyloid deposition present on them. Nevertheless, at this point we cannot rule out the possibility that additional differences in the composition of the AD brains used in this experiment (e.g., differential inflammatory response, variable $A \beta_{40} / A \beta_{42}$ ratios as suggested in Fig. 1, composition and distribution of 


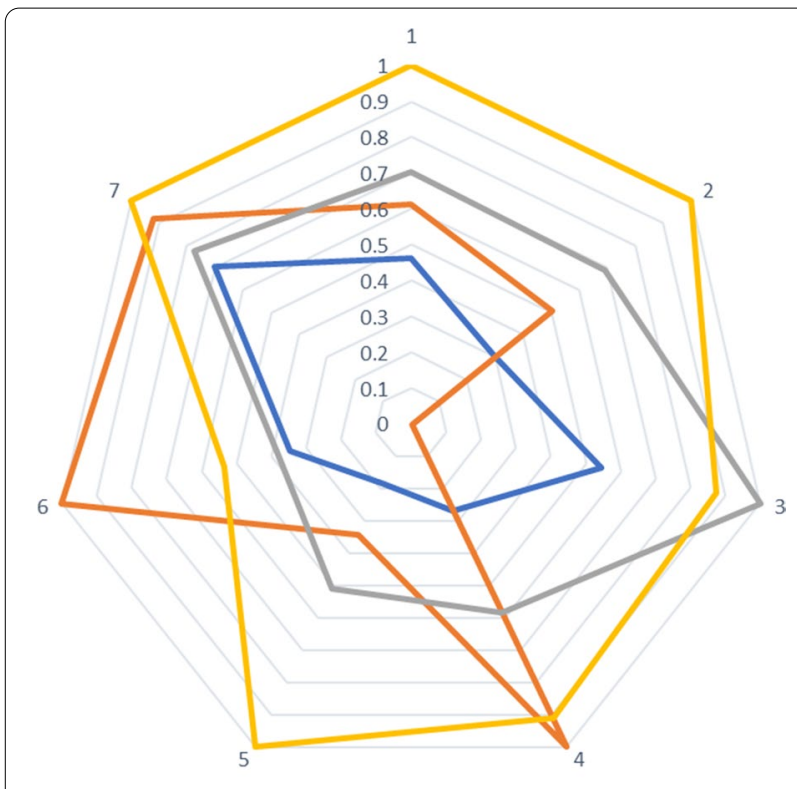

$\longrightarrow$ AD $60129-A D 60068 \longrightarrow$ AD $60649-A D 51486$

Fig. 5 Diagrams depicting pathological features generated by each $A D$ inocula. Each parameter measured in AD brain treated mice were normalized to the higher value among all inocula. These parameters include 4G8 staining in cortex (1), 4G8 staining in hippocampus (2), vascular amyloid deposition (3), ThS staining in cortex (4), ThS staining in hippocampus (5), ThS/4G8 staining in cortex (6) and ThS/4G8 staining in hippocampus (7)

tau pathology, etc.) are responsible for the differences in in vivo seeding. The role of these potential variables in the propagation of $A \beta$ misfolding should be carefully addressed in future studies.

As expected, mice inoculated with the brain extract from an aged non-demented individual displayed the same amount of amyloid deposits compared to noninjected controls (Fig. 2). Nevertheless, a small but significant induction of CAA was observed in mice treated with this particular injectate (Fig. 3). As extensively reported, amyloid deposition is a common event occurring during aging and, in many cases, extensive cerebral amyloid deposition is observed in the absence of clinical signs [60]. As shown in Fig. 1, this "control" brain contains measurable levels of PBS-insoluble A $\beta$ that were actually comparable to the ones measured in one ADderived sample (AD60068). Importantly, these A $\beta$ aggregates were unable to induce substantial pathology, in line with the absence of clinical symptoms observed in this individual.

In the prion field, prion strain selection and cloning require serial infectivity passages within the same animal species $[30,61,62]$. Assuming that misfolded $A \beta$ is also capable to generate conformational strains, similar approaches could be applied to this AD-linked protein. Nevertheless, pathological induction (seeding efficiencies, vascular tropism, ThS reactivity of the aggregates) in this experiment did not resemble the one observed in the parental brains. This could be explained by the aggressive pathology displayed by APP/PS1 due to the introduction of double AD-linked mutations and APP overexpression [44]. In that sense, we believe that the pathology generated in experimental mice is a combined result of seed-templated deposition and the endogenous pathology generated in them. In that sense, the serial in vivo passage approach used to isolate strains of infectious prions may not apply to APP/PS1 mice. In addition, it is predicted that each $\mathrm{AD}$ brain contains not just one, but a combination of different $\mathrm{A} \beta$ strains similarly as it has been hypothesized for their prion counterparts [63]. Future research, using less aggressive models of $A \beta$ deposition and isolated $A \beta$ strains should provide a clearer picture on the role of $A \beta$ conformational strain variation in $\mathrm{AD}$.

One limitation of this study is that estimations of seeding activity were not directly performed by injecting the same amount of $A \beta$ peptides but the same mass of brain tissue. Comparisons of seeding efficiency for each inoculum were done indirectly by calculating the ratio between amyloid induction and the estimated amount of $A \beta$ present in each inoculum. Unfortunately, proper comparisons would need to "titrate" the seeding activity of each material by performing seeding experiments with serial dilutions of each injectate as previously described [24]. This will allow us to assess the effect of $A \beta$ and other potential factors present in the brain that could alter the prion-like propagation of these aggregates (e.g., molecules stabilizing $A \beta$ aggregates, inflammatory components making mice's brains more susceptible for seeding, etc.). In addition, considering that possible misfolded $A \beta$ strains present in each $\mathrm{AD}$ brain are not a single but a group of different co-existing conformations, diluting each brain extract to normalize $\mathrm{A} \beta$ concentrations may not be accurate. Future experiments exploring the composition, stability and biological function of individual and grouped $\mathrm{A} \beta$ strains co-existing in $\mathrm{AD}$ brains could provide us with a better understanding of the biological relevance of the $\mathrm{A} \beta$ conformational strain phenomenon in $\mathrm{AD}$.

\section{Conclusions}

Our results support the hypothesis that AD includes a spectrum of pathological conditions characterized by the accumulation of misfolded proteins leading to dementia. The identification of $A \beta$ strains could lead to personalized and more effective treatments that may improve AD prognosis. 


\section{Supplementary Information}

The online version contains supplementary material available at https://doi. org/10.1186/s40478-021-01155-0.

\section{Additional file 1 .}

\section{Additional file 2 .}

\section{Acknowledgements}

We acknowledge the use of tissues procured by the National Disease Research Interchange (NDRI) with support from NIH grant U42OD11158.

\section{Authors' contributions}

CDA and IM performed most of the experiments, analyzed the data, prepared the figures and reviewed the manuscript. NG and NPU performed staining assessing vascular amyloidosis. LVG contributed to analyze amyloid deposition in the hippocampus. CS participated in study design and data analysis. RM designed the experiment, performed some of the experiments, analyzed the data and wrote the manuscript. All authors read and approved the final manuscript.

\section{Funding}

This work was supported by a grants from the NIH (R56AG061878 and RF1AG059321) to RM and CS, the Alzheimer's Association (AARGD-18-566576 to RM and 2018-AARG-591107 to CDA), ANID/FONDEF ID20110152 and ANID/ FONDECYT 1210622 to CDA, and PID2019-107090RA-100, 275652018 NARSAD and RYC-2017-21879 to IMG.

\section{Availability of data and materials}

The datasets used and/or analyzed during the current study are available from the corresponding author on reasonable request.

\section{Declarations}

\section{Ethics approval and consent to participate}

Brain tissue from AD patients and non-demented individuals were used in this study. Tissues were obtained from the National Disease Research Interchange (NDRI, Philadelphia, PA) in a de-identified manner. Research on human samples was performed following The Code of Ethics of the World Medical Association (Declaration of Helsinki). Informed consent was obtained for human subjects' experimentation. Brain tissues were manipulated following the universal precautions for working with human samples and as directed by the Institutional Review Board (IRB) and the Biosafety Office of The University of Texas Health Science Center at Houston. All animal manipulations were in agreement with $\mathrm{NIH}$ guidelines and approved by the Institutional Animal Care and Use Committee (IACUC) of The University of Texas Health Science Center at Houston.

\section{Consent for publication}

Not applicable.

\section{Competing interests}

The authors declare that they have no competing interests.

\begin{abstract}
Author details
1 Department of Neurology, The University of Texas Health Science Center at Houston, 6431 Fannin, St. Houston, TX 77030, USA. ${ }^{2}$ Center for Social and Cognitive Neuroscience (CSCN), School of Psychology, Universidad Adolfo Ibanez, Santiago, Chile. ${ }^{3}$ Latin American Brain Health Institute (BrainLat), Universidad Adolfo Ibanez, Santiago, Chile. ${ }^{4}$ Universidad de los Andes, Facultad de Medicina, Av. San Carlos de Apoquindo, 2200 Las Condes, Santiago, Chile. ${ }^{5}$ Department of Cell Biology, Faculty of Sciences, University of Malaga-IBIMA, 29010 Malaga, Spain. ${ }^{6}$ Networking Research Center On Neurodegenerative Diseases (CIBERNED), Madrid, Spain. ${ }^{7}$ Centro Integrativo de Biologia Y Quimica Aplicada (CIBQA), Universidad Bernardo O'Higgins, Santiago, Chile.
\end{abstract}

Received: 27 December 2020 Accepted: 14 March 2021 Published online: 30 March 2021

\section{References}

1. Mrcpsych B, Jones E, Ballard C, Gauthier S, Corbett A, Brayne C et al (2011) Alzheimer's disease. Lancet 377:1019-1031

2. Querfurth HW, LaFerla FM (2010) Alzheimer's Disease. N Engl J Med 362:329-344

3. Serrano-Pozo A, Frosch MP, Masliah E, Hyman BT (2011) Neuropathological alterations in Alzheimer disease. Cold Spring Harb Perspect Med, 1

4. Goate A, Chartier-Harlin MC, Mullan M, Brown J, Crawford F, Fidani L et al (1991) Segregation of a missense mutation in the amyloid precursor protein gene with familial Alzheimer's disease. Nature 349:704-706

5. Sherrington R, Rogaev El, Liang Y, Rogaeva EA, Levesque G, Ikeda M et al (1995) Cloning of a gene bearing missense mutations in early-onset familial Alzheimer's disease. Nature 375:754-760

6. Levy-Lahad E, Wasco W, Poorkaj P, Romano DM, Oshima J, Pettingell WH, et al (1995) Candidate gene for the chromosome 1 familial Alzheimer's disease locus. Science (80- ) 269: 973-977

7. Hardy J, Selkoe DJ (2002) The amyloid hypothesis of Alzheimer's disease: progress and problems on the road to therapeutics. Science 297:353-356

8. Krstic D, Knuesel I (2013) Deciphering the mechanism underlying lateonset Alzheimer disease. Nat. Rev, Neurol

9. McGeer PL, McGeer EG (2007) NSAIDs and Alzheimer disease: epidemiological, animal model and clinical studies. Neurobiol. Aging

10. Lam B, Masellis M, Freedman M, Stuss DT, Black SE (2013) Clinical, imaging, and pathological heterogeneity of the Alzheimer's disease syndrome. Alzheimer's Res. Ther

11. Thal DR, Rüb U, Orantes M, Braak H (2002) Phases of Aß-deposition in the human brain and its relevance for the development of AD. Neurology

12. Calderon-Garcidueñas AL, Duyckaerts C (2018) Alzheimer disease. Handb Clin Neurol

13. Duyckaerts C, Delatour B, Potier MC (2009) Classification and basic pathology of Alzheimer disease. Acta Neuropathol 118:5-36

14. Thal DR, Walter J, Saido TC, Fändrich M (2015) Neuropathology and biochemistry of $A B$ and its aggregates in Alzheimer's disease. Acta Neuropathol

15. Lau HHC, Ingelsson M, Watts JC (2020) The existence of A $\beta$ strains and their potential for driving phenotypic heterogeneity in Alzheimer's disease. Acta Neuropathol

16. Galton CJ, Patterson K, Xuereb JH, Hodges JR (2000) Atypical and typical presentations of Alzheimer's disease: a clinical, neuropsychological, neuroimaging and pathological study of 13 cases. Brain

17. Ferreira D, Nordberg A, Westman E (2020) Biological subtypes of Alzheimer disease: A systematic review and meta-analysis. Neurology

18. Gomez-Gutierrez R, Morales R (2020) The prion-like phenomenon in Alzheimer's disease: evidence of pathology transmission in humans. PLoS Pathog

19. Walker LC, Jucker M (2015) Neurodegenerative diseases: expanding the prion concept. Annu Rev Neurosci 38:87-103

20. Soto C (2012) Transmissible proteins: expanding the prion heresy. Cell 149:968-977

21. Soto C, Estrada L, Castilla J (2006) Amyloids, prions and the inherent infectious nature of misfolded protein aggregates. Trends Biochem Sci 31:150-155

22. Duran-Aniotz C, Morales R, Moreno-Gonzalez I, Hu PP, Fedynyshyn J, Soto C (2014) Aggregate-depleted brain fails to induce abeta deposition in a mouse model of Alzheimer's disease. PLoS ONE 9:e89014

23. Meyer-Luehmann M, Coomaraswamy J, Bolmont T, Kaeser S, Schaefer C, Kilger $E$, et al (2006) Exogenous induction of cerebral beta-amyloidogenesis is governed by agent and host. Science (80-) 313:1781-1784

24. Morales R, Bravo-Alegria J, Duran-Aniotz C, Soto C (2015) Titration of biologically active amyloid- $\beta$ seeds in a transgenic mouse model of Alzheimer's disease. Sci Rep 5:9349

25. Eisele YS, Obermüller U, Heilbronner G, Baumann F, Kaeser SA, Wolburg $\mathrm{H}$, et al (2010) Peripherally applied Abeta-containing inoculates induce cerebral beta-amyloidosis. Science (80-) 330: 980-982

26. Morales R, Duran-Aniotz C, Bravo-Alegria J, Estrada LD, Shahnawaz M, Hu PP, Kramm C, Morales-Scheihing D, Urayama A, Soto C (2020) Infusion of blood from mice displaying cerebral amyloidosis accelerates amyloid pathology in animal models of Alzheimer's disease. Acta Neuropathol Commun 8:213 
27. Burwinkel M, Lutzenberger M, Heppner FL, Schulz-Schaeffer W, Baier M (2018) Intravenous injection of beta-amyloid seeds promotes cerebral amyloid angiopathy (CAA). Acta Neuropathol Commun

28. Aguzzi A (1998) Protein conformation dictates prion strain. Nat Med 4:1125-1126

29. Morales R (2017) Prion strains in mammals: different conformations leading to disease. PLoS Pathog

30. Bartz JC, Bessen RA, McKenzie D, Marsh RF, Aiken JM (2000) Adaptation and selection of prion protein strain conformations following interspecies transmission of transmissible mink encephalopathy. J Virol 74:5542-5547

31. Morales R, Hu PP, Duran-Aniotz C, Moda F, Diaz-Espinoza R, Chen B, et al (2016) Strain-dependent profile of misfolded prion protein aggregates. Sci Rep

32. Wadsworth JDF, Collinge J (2011) Molecular pathology of human prion disease. Acta Neuropathol 121:69-77

33. Gambetti P, Kong Q, Zou W, Parchi P, Chen SG (2003) Sporadic and familial CJD: classification and characterisation. Br Med Bull 66:213-239

34. Parchi P, Gambetti P (1995) Human prion diseases. Curr Opin Neurol 8:286-293

35. Morales R, Hu PP, Duran-Aniotz C, Moda F, Diaz-Espinoza R, Chen B et al (2016) Strain-dependent profile of misfolded prion protein aggregates. Sci Rep 6:20526

36. Hu PP, Morales R, Duran-Aniotz C, Moreno-Gonzalez I, Khan U, Soto C (2016) Role of prion replication in the strain-dependent brain regional distribution of prions. J Biol Chem

37. Fraser H, Dickinson AG (1968) The sequential development of the brain lesion of scrapie in three strains of mice. J Comp Pathol 78:301-311

38. Morales R, Callegari K, Soto C (2015) Prion-like features of misfolded A $\beta$ and tau aggregates. Virus Res 207:106-112

39. Watts JC, Condello C, Stöhr J, Oehler A, Lee J, DeArmond SJ et al (2014) Serial propagation of distinct strains of $A \beta$ prions from Alzheimer's disease patients. Proc Natl Acad Sci USA 111:10323-10328

40. Stöhr J, Condello C, Watts JC, Bloch L, Oehler A, Nick M et al (2014) Distinct synthetic $A \beta$ prion strains producing different amyloid deposits in bigenic mice. Proc Natl Acad Sci USA 111:10329-10334

41. Condello C, Lemmin T, Stöhr J, Nick M, Wu Y, Maxwell AM, et al (2018) Structural heterogeneity and intersubject variability of $A \beta$ in familial and sporadic Alzheimer's disease. Proc Natl Acad Sci USA

42. Heilbronner G, Eisele YS, Langer F, Kaeser SA, Novotny R, Nagarathinam A, et al (2013) Seeded strain-like transmission of $\beta$-amyloid morphotypes in APP transgenic mice. EMBO Rep

43. Cohen ML, Kim C, Haldiman T, ElHag M, Mehndiratta P, Pichet T et al (2015) Rapidly progressive Alzheimer's disease features distinct structures of amyloid- $\beta$. Brain 138:1009-1022

44. Jankowsky JL, Fadale DJ, Anderson J, Xu GM, Gonzales V, Jenkins NA et al (2004) Mutant presenilins specifically elevate the levels of the 42 residue beta-amyloid peptide in vivo: evidence for augmentation of a 42-specific gamma secretase. HumMolGenet 13:159-170

45. Wilcock DM, Gordon MN, Morgan D (2006) Quantification of cerebral amyloid angiopathy and parenchymal amyloid plaques with Congo red histochemical stain. Nat Protoc
46. Duran-Aniotz C, Morales R, Moreno-Gonzalez I, Hu PP, Soto C (2013) Brains from non-Alzheimer's individuals containing amyloid deposits accelerate Abeta deposition in vivo. Acta NeuropatholCommun 1:76

47. Duran-Aniotz C, Cornejo VH, Espinoza S, Ardiles ÁO, Medinas DB, Salazar C et al (2017) IRE1 signaling exacerbates Alzheimer's disease pathogenesis. Acta Neuropathol 134:489-506

48. Kelényi G (1967) Thioflavin S fluorescent and congo red anisotropic stainings in the histologic demonstration of amyloid. Acta Neuropathol

49. Duran-Aniotz C, Morales R, Moreno-Gonzalez I, Hu PP, Fedynyshyn J, Soto $C$ (2014) Aggregate-depleted brain fails to induce $A \beta$ deposition in a mouse model of Alzheimer's disease. PLoS ONE 9:e89014

50. Jellinger K (1977) Cerebrovascular amyloidosis with cerebral hemorrhage. JNeurol 214:195-206

51. Yamada M (2013) Brain hemorrhages in cerebral amyloid angiopathy. Semin Thromb Hemost

52. Eisele YS, Fritschi SK, Hamaguchi T, Obermuller U, Fuger P, Skodras A et al (2014) Multiple factors contribute to the peripheral induction of cerebral beta-amyloidosis. JNeurosci 34:10264-10273

53. Aguzzi A, Heikenwalder M, Polymenidou M. Insights into prion strains and neurotoxicity. Nat. Rev. Mol. Cell Biol. 2007. p. 552-61.

54. Bruce ME, Fraser H (1991) Scrapie strain variation and its implications. Curr Top Microbiol Immunol 172:125-138

55. Castilla J, Morales R, Saá P, Barria M, Gambetti P, Soto C (2008) Cell-free propagation of prion strains. EMBO J 27:2557-2566

56. Notari S, Capellari S, Langeveld J, Giese A, Strammiello R, Gambetti P et al (2007) A refined method for molecular typing reveals that co-occurrence of $\operatorname{PrP}(\mathrm{Sc})$ types in Creutzfeldt-Jakob disease is not the rule. Lab Invest 87:1103-1112

57. Bessen RA, Marsh RF (1992) Biochemical and physical properties of the prion protein from two strains of the transmissible mink encephalopathy agent. J Virol 66:2096-2101

58. Qiang W, Yau WM, Lu JX, Collinge J, Tycko R (2017) Structural variation in amyloid- $\beta$ fibrils from Alzheimer's disease clinical subtypes. Nature

59. Langer F, Eisele YS, Fritschi SK, Staufenbiel M, Walker LC, Jucker M (2011) Soluble Abeta seeds are potent inducers of cerebral beta-amyloid deposition. JNeurosci 31:14488-14495

60. Zolochevska O, Taglialatela G (2016) Non-demented individuals with Alzheimer's disease neuropathology: resistance to cognitive decline may reveal new treatment strategies. Curr Pharm Des

61. Morales R, Abid K, Soto C (2007) The prion strain phenomenon: molecular basis and unprecedented features. Biochim Biophys Acta 1772:681-691

62. Lasmézas Cl, Deslys JP, Robain O, Jaegly A, Beringue V, Peyrin JM, et al (1997) Transmission of the BSE agent to mice in the absence of detectable abnormal prion protein. Science (80- ) 275:402-405

63. Collinge J, Clarke AR (2007) A general model of prion strains and their pathogenicity. Science (80-) 318:930-936

\section{Publisher's Note}

Springer Nature remains neutral with regard to jurisdictional claims in published maps and institutional affiliations.

\footnotetext{
Ready to submit your research? Choose BMC and benefit from:

- fast, convenient online submission

- thorough peer review by experienced researchers in your field

- rapid publication on acceptance

- support for research data, including large and complex data types

- gold Open Access which fosters wider collaboration and increased citations

- maximum visibility for your research: over 100M website views per year
}

At BMC, research is always in progress.

Learn more biomedcentral.com/submissions 